\title{
Effect of COVID-19 Epidemic on Delay of Diagnosis and Treatment Path for Patients with Nasopharyngeal Carcinoma
}

This article was published in the following Dove Press journal: Cancer Management and Research

\section{Youqi Yang \\ Chunying Shen \\ Chaosu $\mathrm{Hu}$}

Department of Radiation Oncology, Fudan University Shanghai Cancer Center, Department of Oncology, Shanghai Medical College, Shanghai 200032, People's Republic of China

Correspondence: Chaosu $\mathrm{Hu}$ No. 270, Dong'an Road, Shanghai, People's Republic of China TelFax + 8602164I75590

Email hucsu62@yahoo.com
Introduction: 2019 novel coronavirus disease (COVID-19) outbreaks have been occurring in China and other countries in the world. To prevent further spread of the disease, restrictions of population flow from the government and measures to reduce virus transmission from hospitals may lead to the delay of diagnosis and treatment in patients with nasopharyngeal carcinoma (NPC).

Methods: All NPC patients with radiotherapy indications were included from 20 weekdays before (group A) and after (group B) January 31, 2020, when the institute began to take measures against COVID-19. The waiting intervals of each step and variation from the diagnosis and treatment path of NPC between two groups were compared.

Results: Significant differences were found between the group A and group B in the median waiting days for pathological biopsy ( 5 vs $15, P=0.012$ ), radiotherapy immobilization and simulation (3.5 vs $16.5, P<0.001)$, validation of position and plan $(20 \mathrm{vs} 61, P<0.001)$ and initiation of radiotherapy ( 28 vs $36, P=0.005$ ). During the waiting period of radiotherapy, $32.4 \%$ of the NPC patients received an additional one cycle of chemotherapy to the original treatment strategy.

Conclusion: The prevalence of COVID-19 caused delay in the diagnosis and treatment of NPC patients to a certain extent. Additional chemotherapy could be considered to counteract the effect of treatment delay. More specific measures should be taken to balance the risk of delayed diagnosis and treatment of NPC and infection of COVID-19.

Keywords: COVID-19, nasopharyngeal carcinoma, radiotherapy

\section{Introduction}

Since the outbreak of epidemic outbreak of coronavirus disease 2019 (COVID-19) in China, ${ }^{1}$ Chinese government has taken effective isolation measures to prevent further spread of the disease. With the lockdown of Wuhan announced, areas outside Hubei Province in China also proposed strict measures, including home quarantine for at least 14 days, social distancing, and postponing of work resumption to January 31, 2020. Medical centers outside Hubei Province in China strengthened the monitoring and screening of imported asymptomatic cases $^{2}$ to avoid potential hospital outbreak of COVID-19. For the non-emergency patients without symptoms of fever, cough, nasal catarrh, chest pain, muscle ache, joint pain, shortness of breath or diarrhea from 22 epidemic provinces, autonomous regions and municipalities, a minimum 14 days of medical observation was required before hospitalization after leaving the epidemic areas. It is noteworthy that in China, many patients would go to big cities such as 
Beijing, Shanghai and Guangzhou seeking for diagnosis and treatment of malignancies. Given the long course of antitumor treatments, especially radiotherapy and chemotherapy, multiple roundtrips between cities are usually inevitable for patients. Due to the restrictions on the intercity flow of population and hospital admissions, the diagnosis and treatment of cancer patients might be hindered to some extent.

The conventional treatment methods of nasopharyngeal carcinoma (NPC) include radiotherapy and systemic treatment. It is generally recommended that chemotherapy and radiotherapy are given on time for patients with locally advanced tumor. There is a correlation between radiotherapy postponing and worsened clinical outcome. ${ }^{3-6}$ Considering that the outbreak of COVID-19 may delay the diagnosis and treatment of NPC patients, this article attempts to compare the efficiency of clinical workflow in an NPC cohort from a single cancer center within 20 weekdays before and after January 31, 2020, when institutional anti-COVID-19 measures activated.

\section{Materials and Methods}

\section{Patients Enrollment}

A total of 874 patients who visited clinics in head and neck department were identified from database of Fudan University Shanghai Cancer Center between December 27, 2019 and February 26, 2020. The criteria for inclusion were as follows: (i) histologically confirmed NPC; (ii) patients with indication of radiotherapy who entered the path of diagnosis and treatment in our hospital. The patients who received radiotherapy elsewhere or had finished radiotherapy before this period were excluded.

\section{Clinical Management Pathway and Data Collection}

The path of diagnosis and treatment for NPC included pathological consultation or biopsy, imaging examination, radiotherapy immobilization and simulation, validation of position and plan, and initiation of radiotherapy. The data of all outpatient visits, average length of stay in hospital, turnover ratio of beds and number of NPC patients undergoing radiation in 20 weekdays before and after January 31 , 2020 were collected from the medical documents.

\section{Data Analysis}

Outbreak in this study is defined as the date when the government takes isolation and restriction measures, and the hospital takes measures to prevent nosocomial infection of COVID-19, ie, January 31, 2020. This cohort was divided into two groups according to the date when patients entered each step of the path: from December 27, 2019 to January 23, 2020 (group A), and from January 31, 2020 to February 26, 2020 (group B). It compared the waiting intervals in the diagnosis and treatment path of NPC and variation from the standard treatment since the COVID-19 outbreak. The baseline characteristics of the two groups were compared, including gender, age, stage (according to 8th edition American Joint Committee on Cancer staging system), chemotherapy, Karnofsky performance status (KPS), comorbidities and patients from other cities. The definition of comorbidities includes any known medical diseases that may extend the waiting time in the pathway, including hypertension, diabetes, atrial fibrillation and so on, and chemotherapy-related adverse reactions, such as myelosuppression above grade III, liver and kidney dysfunction above grade II according to Common Terminology Criteria for Adverse Events (Version 4.0). The waiting time of each link in the path of the two groups was compared. Except for the definition of initiation of radiotherapy as the last treatment or pathological diagnosis to the start of radiotherapy, the waiting time for other links are sequential.

\section{Statistical Methods}

We performed chi-square and $t$-test on the baseline clinical characteristics, waiting time and variation in the pathway. A two-sided $P$-value was calculated and $P<0.05$ was considered significant. All the statistical analyses were used by SPSS 19 .

\section{Results}

From the perspective of hospital management, from December 27, 2019 to January 23, 2020, the number of outpatient visits was 914 in 20 weekdays. From January 31, 2020 to February 26, the number of outpatient visits was 266 in 20 weekdays. The average length of stay of discharged patients in the two periods was 11.5 days and 25 days, respectively, and the bed turnover rate was 2.1 and 0.5 , respectively. The number of patients undergoing radiotherapy was 52 and 24, respectively.

From 874 cases during the period, 738 patients were excluded from the cohort including 394 non-NPC patients, 332 post-treatment NPC patients for follow-up or recurrence or distant metastasis, and 12 NPC patients who chose to receive treatment elsewhere. 
A total of 136 patients with NPC had indications for radiotherapy and entered the diagnosis and treatment path. There were 112 patients in group A and 82 in group B. The number of cases waiting for pathological consultation report, pathological biopsy report, imaging examination, immobilization and simulation, validation of position and plan, radiotherapy and undergoing radiotherapy in the two groups were recorded in the flow chart of Figure 1. Because of the long waiting time of each step for radiotherapy preparations, the cases from two groups entering the path were partly overlapped. For example, a patient in group A waiting for pathological report was waiting for radiotherapy in the path of group B. The patient was included in the analysis of waiting interval in different phases of two groups, because of the different time point of each step entering the diagnosis and treatment path. There were 58 patients overlapped in the two groups. The baseline characteristics of the two groups were well-balanced (Table 1).

The waiting time in each step of the path from two groups was recorded and compared (Table 2). From the patients' perspective, the median waiting time of pathological consultation report before and after January 31, 2020, was 3 and 2 days, respectively. The median waiting time for pathological reports directly from biopsies by nasopharyngoscopy in our hospital was 5 days before the outbreak. For imaging examinations such as MRI, CT, etc., the median appointment time in group A and group B was 8 days and 1 day, respectively. The median waiting time of radiotherapy immobilization and simulation before the outbreak was 3.5 days. After the outbreak, the median waiting time was 16.5 days. The median waiting intervals of radiotherapy validation of position and plan were 20 and 61 days, respectively. And the median waiting time of radiotherapy was 28 and 36 days, respectively, with statistically significant differences.

In our center, patients with stage I cancer were treated with definitive radiotherapy alone. For patients with stage II-IVa, induction chemotherapy followed by radiotherapy or concurrent chemoradiotherapy were considered as treatment options. Patients with stage $\mathrm{IVb}$ received 4-6 cycles of chemotherapy followed by radiotherapy. There were 39 patients in group B waiting for radiotherapy with completed radiation treatment planning. Of the 39 patients, 34 received chemotherapy, 1 refused chemotherapy, and 4 did not need chemotherapy. Among the 34 patients who received neoadjuvant chemotherapy, 11 patients (32.4\%) added one cycle of chemotherapy to the original treatment strategy due to delayed radiotherapy. For the patients who should be given concurrent chemoradiotherapy according to the original treatment schedule, the completion rate of chemotherapy within 1 month before and after the outbreak of the epidemic was $84.6-75 \%$, respectively (Table 3 ).

\section{Discussion}

Since the outbreak of COVID-19 in China, the medical care system has been profoundly affected by cancer

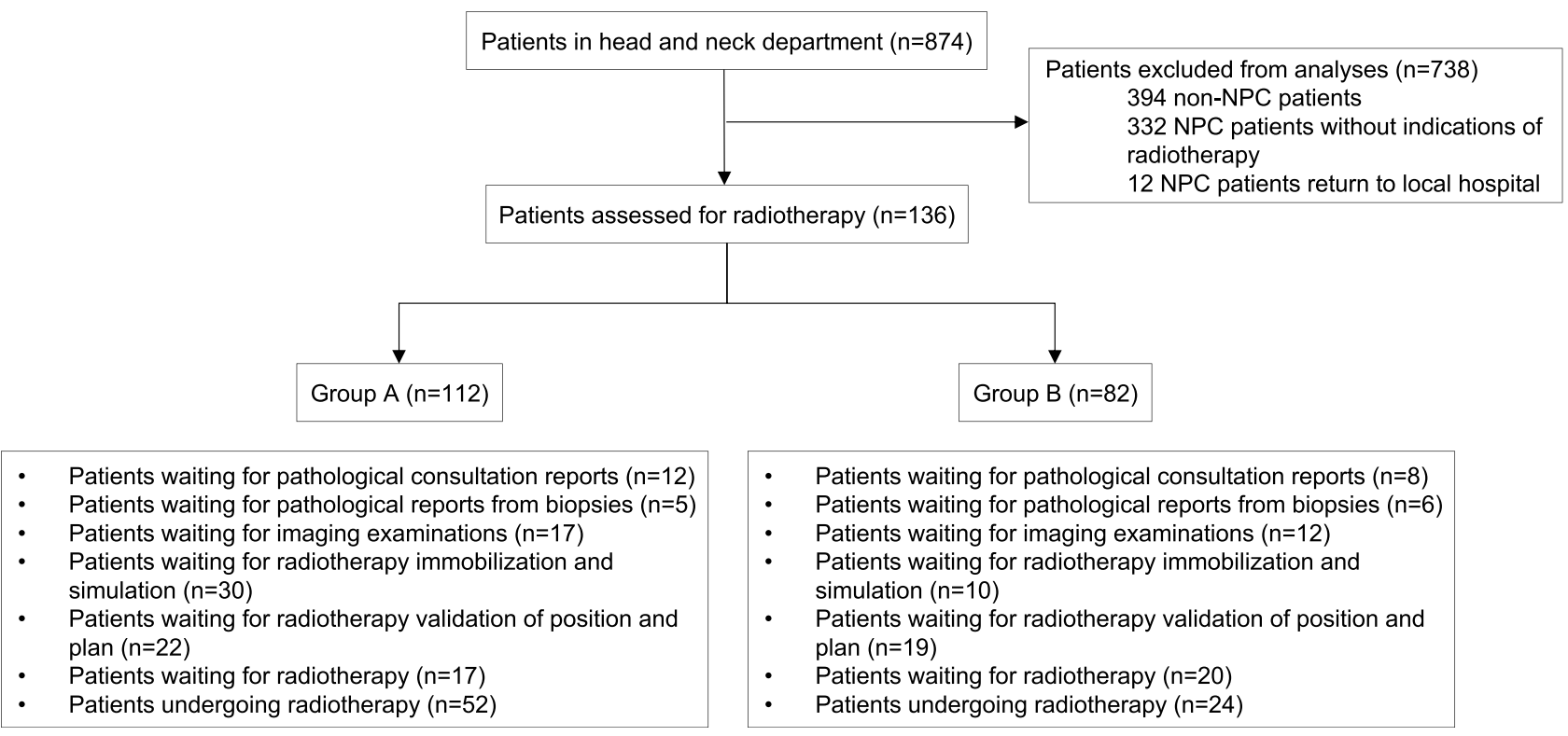

Figure I Flow chart of NPC patients eligible for radiotherapy. Group A: patients entered diagnosis and treatment path of NPC from December 27, 2019 to January 23, 2020 $(n=\mid 12)$. Group B: patients entered diagnosis and treatment path of NPC from January 31, 2020 to February $26(n=82)$. 
Table I Baseline Characteristics of Patients Enrolled Before (Group A) and After (Group B) Control Strategy of COVID- 19 Epidemic

\begin{tabular}{|l|l|l|l|}
\hline Characteristic & Group A & Group B & P value \\
\hline $\begin{array}{l}\text { Gender n (\%) } \\
\text { Male } \\
\text { Female }\end{array}$ & $\begin{array}{l}84(75.0) \\
28(25.0)\end{array}$ & $\begin{array}{l}60(73.2) \\
22(26.8)\end{array}$ & 0.774 \\
\hline $\begin{array}{l}\text { Age(years) } \\
\text { Median(range) }\end{array}$ & $50.5(18-77)$ & $50.5(18-7 \mid)$ & \\
\hline $\begin{array}{l}\text { Stage } \mathrm{n}(\%) \\
\text { I }\end{array}$ & $5(4.5)$ & $4(4.9)$ & 0.606 \\
II & $10(8.9)$ & $5(6.1)$ & \\
III & $42(37.5)$ & $35(42.7)$ & \\
IVa & $4 I(36.6)$ & $31(37.8)$ & \\
IVb & $14(12.5)$ & $7(8.5)$ & \\
\hline Chemotherapy received $\mathrm{n}(\%)$ & $100(89.3)$ & $74(90.2)$ & 0.828 \\
\hline $\begin{array}{l}\text { Karnofsky performance status } \\
\mathrm{n}(\%)\end{array}$ & & & 0.446 \\
70 & $2(1.8)$ & $0(0)$ & \\
80 & $3(2.7)$ & $3(3.7)$ & \\
90 & $107(95.5)$ & $79(96.3)$ & \\
\hline $\begin{array}{l}\text { Comorbidities n(\%) } \\
\text { Patients from other cities } \mathrm{n}(\%)\end{array}$ & $32(28.6)$ & $23(28.0)$ & 0.936 \\
\hline
\end{tabular}

Abbreviation: COVID-19, 2019 novel coronavirus disease.

patients, including those with NPC. Compared with that within 20 weekdays before the launch of the epidemic control policies, the number of outpatient visits after January 31, 2020, decreased significantly. Most of the patients from other provinces were prone to be affected by traffic restrictions. Patients from epidemic areas had to take a medical observation for 14 days after arriving in new cities. As a result, a considerable part of patients failed to arrive at hospital on time, leading to a significantly decreased the number of outpatient visits, which was subsequently followed by shrunken number of inpatients, extended average length of stay as well as reduced bed turnover rate. The number of patients
Table 3 Variation of Diagnosis and Treatment Path of NPC Under the Controlling Measure of the COVID-19

\begin{tabular}{|l|l|l|l|}
\hline Variation of Events & $\begin{array}{l}\text { Group A } \\
\mathbf{n}(\%)\end{array}$ & $\begin{array}{l}\text { Group B } \\
\mathbf{n}(\%)\end{array}$ & P value \\
\hline $\begin{array}{l}\text { Additional chemotherapy } \\
\text { received } \\
\begin{array}{l}\text { Concurrent chemotherapy } \\
\text { reduced }\end{array}\end{array}$ & $2(5.9)$ & $\mathrm{II}(32.4)$ & 0.006 \\
\hline
\end{tabular}

Abbreviation: NPC, nasopharyngeal carcinoma.

undergoing radiotherapy also decreased as an effect of less patient visits.

In comparison, the waiting time for pathological consultation and imaging examinations was shorter than before due to decreased patient visits and appointments. Considering that the COVID-19 can spread through droplets or direct contact, ${ }^{7}$ the number of endoscopic operations was strictly limited. There were 6 patients in the outpatient clinics considered to have nasopharyngeal mass, but the biopsy could not be done by endoscopy in time. As a requirement of patient distancing, the capacity of radiotherapy and related work was reduced, so the immobilization and simulation, validation of position and plan, and initiation of radiotherapy were all delayed. Besides, extra effort for COVID-19 screening with a standard workup of epidemiological history inquiry, temperature monitoring and chest $\mathrm{CT}$ added to the waiting time, also leading to the postponing of diagnosis and radiotherapy of NPC.

It has been reported that the delay for anti-tumor treatment in patients with tumor may affect efficacy. It was found that delay in diagnosis correlates with the stage of nasopharyngeal carcinoma. ${ }^{8}$ Sharon et al reported that long diagnose-to-treatment interval to radiotherapy contributes to poor clinical outcome for nasopharyngeal carcinoma patients. ${ }^{3}$ Liang et al also demonstrated that increasing waiting time for radiotherapy beyond 30 days

Table 2 Waiting Interval for Different Stages of Diagnosis and Treatment Path

\begin{tabular}{|l|l|l|l|}
\hline Waiting Interval in Days & $\begin{array}{l}\text { Group A } \\
\text { Median(Range) }\end{array}$ & $\begin{array}{l}\text { Group B } \\
\text { Median(Range) }\end{array}$ & P value \\
\hline Pathological consultation report & $3(2-20)$ & $2(I-3)$ & $0.11 \mathrm{I}$ \\
Pathological reports from biopsies & $5(I-6)$ & $15(5-18)$ & 0.012 \\
Imaging examination appointment & $8(6-12)$ & $\mathrm{I}(0-1)$ & $<0.00 \mathrm{I}$ \\
Radiotherapy immobilization and simulation & $3.5(0-19)$ & $16.5(0-47)$ & $<0.00 \mathrm{I}$ \\
Radiotherapy validation of position and plan & $20(6-33)$ & $6 I(43-8 I)$ & $<0.00 I$ \\
Initiation of radiotherapy & $28(I-34)$ & $36(16-106)$ & 0.005 \\
\hline
\end{tabular}


was detrimental to survival. ${ }^{4}$ Chen et al confirmed that prolonged wait time ( $>4$ weeks) was an independent unfavorable prognostic factor for patients with nasopharyngeal carcinoma. ${ }^{5}$ Another study confirmed similar results. ${ }^{6}$ Based on these evidence, we sought to reduce the waiting time by adding another cycle of induction chemotherapy to the scheduled treatment for those who could not get access to radiotherapy in time. For patients who did not receive increased chemotherapy courses, the median waiting time prior to radiotherapy was significantly longer after COVID-19 outbreak. Telephone contact revealed that the reasons for the lack of compensatory chemotherapy included that local hospitals refused to accept patients who had been treated in other hospitals, patients refused to increase or could not tolerate additional chemotherapy courses. The median waiting time for initiation of radiotherapy is significantly longer since the outbreak.

For patients who had started radiotherapy, considering the aggregation of people in the infusion center and impaired immunity of patients undergoing radiotherapy and chemotherapy, the hospital suggests minimizing intravenous infusion with serious adverse reactions. Patients who received concurrent chemotherapy might experience severe hematologic toxicities and gastrointestinal reactions. ${ }^{9,10}$ So the completion rate of concurrent chemotherapy was lower than before. Liu et al reported that $200 \mathrm{mg} / \mathrm{m}^{2}$ of concurrent cisplatin could be the optimal dose for the NPC patients who achieved response after induction chemotherapy. ${ }^{11}$ Patients receiving higher cumulative dose of cisplatin showed significantly improved 3-year progression-free survival (PFS) and distant metastasis-free survival (DMFS) compared with lower dose patients. Ou et al demonstrated that total dose of cisplatin more than $300 \mathrm{mg} / \mathrm{m}^{2}$ was independent prognostic factors for DMFS, disease-free survival (DFS) and overall survival (OS) in NPC patients. ${ }^{12}$ However, several articles retrospectively reported that IMRT alone achieves similar survival outcomes compared with concurrent chemoradiotherapy in locally advanced NPC patients. ${ }^{13,14}$ In our cohort, whether the reduction of concurrent platinum would detriment patients' outcome will need to be closely followed up in future.

Liang et al, ${ }^{15}$ recently reported that $1 \%$ of 1590 COVID19 cases had a history of cancer, the proportion of COVID-19 infected cancer patients was higher than that of overall cancer incidence rate in Chinese population (0.29\%). Cancer patients had a higher risk of developing severe illness than non-cancer patients (39\% vs $8 \%$ ). Patients who had received chemotherapy in the past month were more likely to get worse (75\% vs 43\%). Therefore, Liang et al suggested that in the epidemic areas, delaying chemotherapy or elective surgery could be considered for stable cancer. However, for the tumor with locally advanced stage, poor dedifferentiation and rapid progression, for example, nasopharyngeal carcinoma, we suggested that the patients should be treated in time, in case the optimal timeframe of treatment is missing. For the patients undergoing chemotherapy, replacement with less toxic chemotherapy agents, reduction of treatment intensity, delaying or omitting concurrent chemotherapy could also be considered, so as to minimize the risk of COVID19 infection. How to make the best choice between timely anti-cancer treatment and avoiding COVID-19 related risk is still worth thinking and further investigation. According to the urgency of anti-tumor treatment, it may be a feasible way to develop differentiated strategies.

The proportion of non-local patients is similar in two groups. As mentioned above, the reasons for the delay of diagnosis and treatment include traffic restrictions. We consider that the countermeasures for traffic restrictions under the epidemic include: (i) to refine the patient management, including using big data of travel to analyze and assess the risk of patients infected with COVID-19, so as to improve the efficiency of diagnosis and treatment; (ii) to reduce the treatment interruption and delay caused by traffic blockade through telemedicine and other technologies; (iii) to strengthen academic cooperation and alliances among hospitals in different regions, so as to reduce the difference of medical level among regions.

There were several limitations of this study. Firstly, selection bias is unavoidable for its retrospective nature, with small samples in a single center. Secondly, this study focuses on the delay in the diagnosis and treatment path. At present, there are no long-term survival follow-up data to fully evaluate the impact of the delay on the prognosis of patients.

\section{Conclusion}

In conclusion, prevention and control measures for the outbreak of COVID-19 have postponed the diagnosis and treatment of patients with NPC, which may affect the prognosis of patients to a certain extent, or increase unnecessary treatment intensity of patients. Timely and effective treatment for patients with high-risk recurrence under the controlling measure of the COVID-19 was needed so as to ensure the efficacy of these patients. And we call for the introduction of guidance related to the diagnosis and treatment of NPC with more detailed supervision to deal 
with the impact of COVID-19 control measures. It is worth noting the efficacy of patients during the epidemic period with long-term follow-up.

\section{Ethics and Consent Statements}

This study was approved by the Ethical Committee and Institutional Review Board of Fudan University Shanghai Cancer Centre (FDUSCC). The method was based on approved guidelines. Informed consent was written by all participants.

\section{Acknowledgments}

We thank all the patients who consented to the study and the anonymous reviewers for their helpful comments.

\section{Disclosure}

The authors report no conflicts of interest in this work.

\section{References}

1. Zhu N, Zhang D, Wang W, et al. A novel coronavirus from patients with pneumonia in China, 2019. N Engl J Med. 2020;382(8):727-733. doi:10.1056/NEJMoa2001017

2. Guan WJ, Ni ZY, Hu Y, et al. Clinical characteristics of coronavirus disease 2019 in China. N Engl J Med. 2020;382:1708-1720. doi:10. 1056/NEJMoa2002032

3. Stoker SD, Wildeman MA, Fles R, et al. A prospective study: current problems in radiotherapy for nasopharyngeal carcinoma in yogyakarta, indonesia. PLoS One. 2014;9(1):e85959. doi:10.1371/journal.pone. 0085959

4. Liang H, Xiang YQ, Lv X, et al. Survival impact of waiting time for radical radiotherapy in nasopharyngeal carcinoma: A large institution-based cohort study from an endemic area. Eur J Cancer. 2017;73:48-60. doi:10.1016/j.ejca.2016.12.009

5. Chen YP, Mao YP, Zhang WN, et al. Prognostic value of wait time in nasopharyngeal carcinoma treated with intensity modulated radiotherapy: a propensity matched analysis. Oncotarget. 2016;7(12):14 973-14982. doi:10.18632/oncotarget.7789
6. Stoker SD, Fles R, Herdini C, et al. The impact of the overall radiotherapy time on clinical outcome of patients with nasopharyngeal carcinoma; a retrospective study. PLoS One. 2016;11(3):e151899. doi:10.1371/journal.pone. 0151899

7. Lai CC, Shih TP, Ko WC, Tang HJ, Hsueh PR. Severe acute respiratory syndrome coronavirus 2 (SARS-CoV-2) and coronavirus disease-2019 (COVID-19): the epidemic and the challenges. Int J Antimicrob Agents. 2020;55:105924. doi:10.1016/j.ijantimicag. 2020.105924

8. Sheng L, Shui Y, Shen L, Wei Q. Effect of patient-related delay in diagnosis on the extent of disease and prognosis in nasopharyngeal carcinoma. Am J Rhinol. 2008;22(3):317-320. doi:10.2500/ajr.2008. 22.3174

9. You R, Sun R, Hua YJ, et al. Cetuximab or nimotuzumab plus intensity-modulated radiotherapy versus cisplatin plus intensity-modulated radiotherapy for stage II-IVb nasopharyngeal carcinoma. Int J Cancer. 2017;141(6):1265-1276. doi:10.1002/ijc.30 819

10. Tang LQ, Chen DP, Guo L, et al. Concurrent chemoradiotherapy with nedaplatin versus cisplatin in stage II-IVB nasopharyngeal carcinoma: an open-label, non-inferiority, randomised Phase 3 trial. Lancet Oncol. 2018;19(4):461-473. doi:10.1016/S1470-2045(18) 30104-9

11. Liu SL, Sun XS, Yan JJ, et al. Optimal cumulative cisplatin dose in nasopharyngeal carcinoma patients based on induction chemotherapy response. Radiother Oncol. 2019;137:83-94. doi:10.1016/j.radonc.20 19.04.020

12. Ou X, Zhou X, Shi Q, et al. Treatment outcomes and late toxicities of 869 patients with nasopharyngeal carcinoma treated with definitive intensity modulated radiation therapy: new insight into the value of total dose of cisplatin and radiation boost. Oncotarget. 2015;6 (35):38381-38397. doi:10.18632/oncotarget.5420

13. Wei Z, Zhang Z, Luo J, Li N, Peng X. Induction chemotherapy plus IMRT alone versus induction chemotherapy plus IMRT-based concurrent chemoradiotherapy in locoregionally advanced nasopharyngeal carcinoma: a retrospective cohort study. $J$ Cancer Res Clin Oncol. 2019;145(7):1857-1864. doi:10.1007/s00432-019-02925-z

14. Chen X, Zhu X, Wang J, Liu J, Ji R. NACT+IMRT versus NACT+IMRT +CCRT in locoregionally advanced NPC patients: a retrospective study. Onco Targets Ther. 2019;12:1553-1562. doi:10.2147/OTT.S183483

15. Liang W, Guan W, Chen R, et al. Cancer patients in SARS-CoV-2 infection: a nationwide analysis in China. Lancet Oncol. 2020;21 (3):335-337. doi:10.1016/S1470-2045(20)30096-6

\section{Publish your work in this journal}

Cancer Management and Research is an international, peer-reviewed open access journal focusing on cancer research and the optimal use of preventative and integrated treatment interventions to achieve improved outcomes, enhanced survival and quality of life for the cancer patient.
The manuscript management system is completely online and includes a very quick and fair peer-review system, which is all easy to use. Visit http://www.dovepress.com/testimonials.php to read real quotes from published authors. 